\title{
ABF STEM Characterization of Light Elements in Ceramic Interface
}

\author{
Y. Ikuhara, ${ }^{* * * * * * *}$ R. Huang, ${ }^{* *}$ S.D. Findlay, ${ }^{*}$ T. Mizoguchi, ${ }^{*}$ N. Shibata, ${ }^{*}$ and T. Hirayama** \\ * Institute of Engineering Innovation, the University of Tokyo, Tokyo 113-8656, Japan \\ ** Nanostructures Research Laboratory, Japan Fine Ceramics Center, Nagoya, 456-8587, Japan \\ *** WPI Advanced Institute for Materials Research, Tohoku University, Sendai, 980-8577, Japan
}

Grain boundaries (GB) of crystals have peculiar electronic structures, caused by the disorder in periodicity, providing the functional properties, which cannot be observed in a perfect crystal. Ceramic materials are composed of light elements, and light elements in the GB structural units play a crucial role in the material properties. Recently we have reported that annular bright field (ABF) STEM imaging is very powerful technique to produce images showing both light and heavy element columns simultaneously [1]. In this technique, an annular detector is located within the bright-field (direct-scattered) region, and the columns display absorptive-type contrast. Importantly, ABF image contrast does not change remarkably over a wide thickness range, which gives a simple and reliable image interpretation. This method thus represents a less time-consuming technique for reliably identifying the presence and positions of light elements. In this study, GB atomic structures including light elements in several ceramics are directly observed by ABF STEM. STEM observations were performed on a Cs-corrected JEM-2100F equipped with Gatan Image Filter (GIF). Fig. 1 shows the ABF STEM image of pristine $\mathrm{Al}_{2} \mathrm{O}_{3} \Sigma 13 \mathrm{~GB}$, which were observed along the $<1 \overline{2}$ $10>$ direction [2]. The image was smoothed by a smoothing filter, and oxygen columns in the GB are clearly visible as well as the oxygen contrast in the bulk. The structural model for the oxygenterminated GB determined by first principles calculations [3] indicates that the oxygen columns are periodically located in the center of the GB structural unit as indicated by the arrows. This model and the simulated ABF STEM image are overlaid on the image, which are good agreement with the experimentally obtained ABF image.

The properties of lithium battery are strongly dependent on the behavior of lithium ions during charge/discharge process. Since this behavior determines the stability, lifetime and reliability, direct visualization of $\mathrm{Li}$ site is needed to understand the mechanism of the properties. ABF STEM technique is demonstrated to directly observe Li and $\mathrm{O}$ ions in lithium battery materials [3,4]. Fig. 2(a) shows $\mathrm{ABF}$ image of the subsurface region of $\mathrm{LiMn}_{2} \mathrm{O}_{4-\delta}$ particle viewed along [100], using a detection angle of 6-25 mrad [3]. The Li ions are clearly visible together with the $\mathrm{O}$ and Mn columns in the ABF image, as indicated by the model structure overlaid in the image. They slightly shift away from their original positions due to the presence of $\mathrm{O}$ vacancies. The image simulation reproduces the image contrast in the experimental ABF image. Fig.2(b) shows a typical ABF image obtained from the $\mathrm{LiCoO}_{2}$ thin film viewed along $<11 \overline{2} 0>$. Li sites as well as $\mathrm{O}$ and Co sites are clearly visible, which can be distinguished according to the image contrast, as illustrated by the atomic structure model inset in the image. These examples demonstrate that ABF imaging in Cscorrected STEM is a reliable technique for directly observing light elements in ceramics. We'll also show that even hydrogen columns can be imaged in several metal hydride by ABF STEM [6] in this presentation. This indicates that ABF techniques in Cs-corrected STEM will be very useful to directly characterize light elements in any materials. 
References

[1] S. D. Findlay et al., Appl. Phys. Lett., 95, 19191 (2009).

[2] S. D. Findlay et al.,Ultramicroscopy, 111, 285 (2011).

[3] S. Azuma et al., Phil. Mag. Lett., 90, 539 (2010).

[4] R. Huang et al., Angew Chem Int. Ed., in press (2011).

[5] R. Huang et al., Appl. Phys. Lett., 98, 051913 (2011).

[6] S.D.Findlay et al., Appl. Phys. Expess, 3, 116603 (2010).

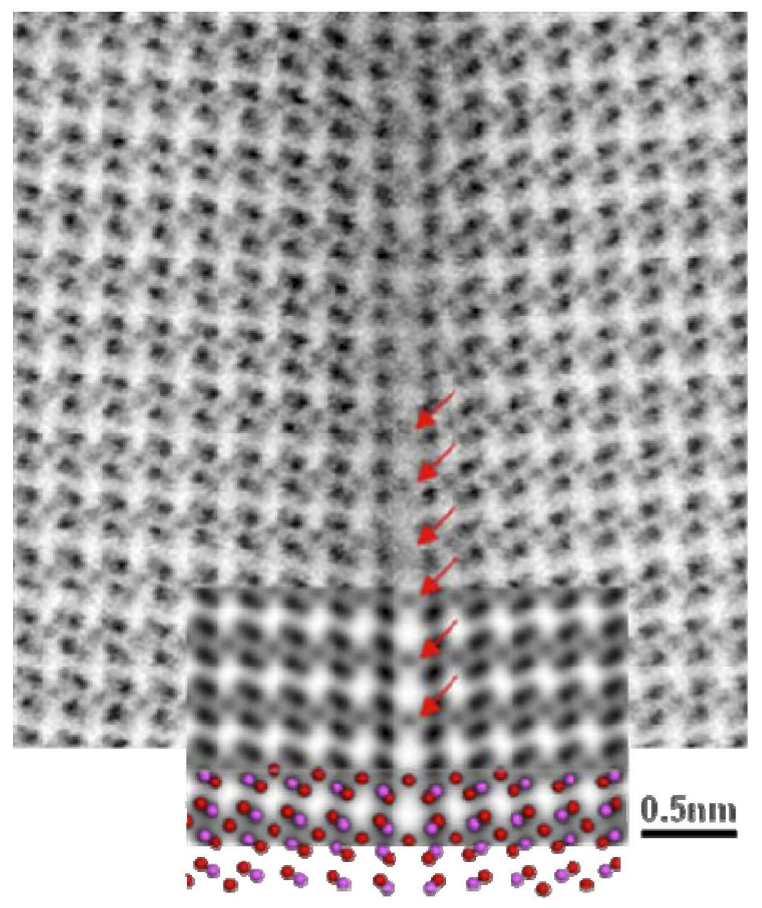

FIG.1 Experimental ABF STEM image of the $\Sigma 13$ (1014) grain boundary in $\alpha-\mathrm{Al}_{2} \mathrm{O}_{3}$ viewed along the $[1210]$ zone axis. The grain boundary atomic structure obtained by the first principles calculation (red:oxygen, pink:aluminum) and the simulated images are overlaid on the experimental image.
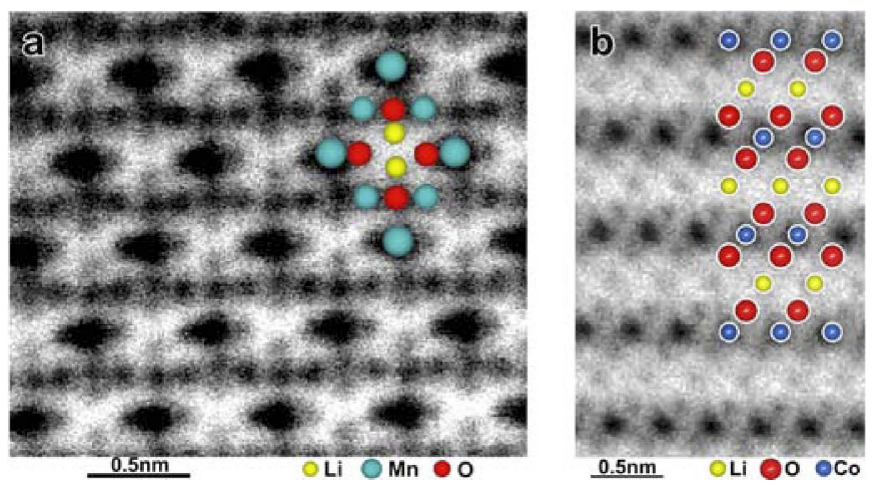

FIG.2 (a) ABF STEM image of tetragonal $\mathrm{LiMn}_{2} \mathrm{O}_{4-\delta}$ spinel nanoparticles along zone axis [100]. (b) A typical ABF image of $\mathrm{LiCoO}_{2}$ thin film viewed along the [1120] zone axis, which shows that the Li columns are clearly visible. Both of the crystal structure models are superposed.

\section{Acknowledgements}

Part of this work was supported This research is supported by the Japan Society for the Promotion of Science (JSPS) through its "Funding Program for World-Leading Innovative R\&D on Science and Techonolgy (FIRST Program)". 